COMMUNICATIONS ARISING Astrophysics

\section{Supernova-remnant origin of cosmic rays?}

$\mathrm{t}$ is thought that Galactic cosmic-ray nuclei are gradually accelerated to high energies (up to about $300 \mathrm{TeV}$ per nucleon, where $1 \mathrm{TeV}$ is $10^{12} \mathrm{eV}$ ) in the expanding shock waves connected with the remnants of powerful supernova explosions. However, this conjecture has eluded direct observational confirmation ${ }^{1,2}$ since it was first proposed in 1953 (ref. 3). Enomoto et al. claim to have finally found definitive evidence that corroborates this model, proposing that very-high-energy, TeV-range, $\gamma$-rays from the supernova remnant (SNR) RX J1713.7-3946 are due to the interactions of energetic nuclei in this region. Here we argue that their claim is not supported by the existing multiwavelength spectrum of this source. The search for the origin(s) of Galactic cosmic-ray nuclei may be closing in on the long-suspected supernova-remnant sources, but it is not yet over.

We have previously suggested ${ }^{5}$ that the SNR RX J1713.7-3946 (dark X-ray contours in Fig. 1) might be an accelerator of cosmic-ray nuclei on the basis of $\mathrm{GeV}\left(10^{9}\right.$ eV) $\gamma$-ray emission ${ }^{6}$ (Fig. 1, white contours) seen towards adjacent massive and dense molecular clouds (Fig. 1, false colour), with which it seems to be interacting ${ }^{7}$; the $\gamma$-ray signature of nucleonic interactions is known to be greatly amplified in such dense media. Enomoto et al. have since claimed ${ }^{4}$ that the $\gamma$-rays of even higher energy $(\mathrm{TeV}$ range) that they detected directly towards the more rarefied remnant (red contours in Fig. 1) are an unambiguous signature of nucleonic interactions in this region, thus finally proving the long-held conjecture.

However, it is well known that SNRs accelerate electrons (see ref. 8, for example) that can also generate $\mathrm{TeV} \gamma$-rays at the source sites. It is therefore generally difficult to assign conclusively an electronic rather than a nucleonic origin to the observed celestial $\gamma$-rays ${ }^{9}$. Fortunately, in this case the discrimination is not so subtle - if the TeV emission seen in the northwest quadrant of the remnant were really due to protons, as proposed $^{4}$, that part of the SNR would have been detected as a bright $\mathrm{GeV}$ source in the Energetic Gamma Ray Experiment Telescope (EGRET) all-sky survey ${ }^{6}$, but it was not.

The proposed model ${ }^{4}$ is unsatisfactory because it predicts that there will be roughly three times the $\mathrm{GeV} \gamma$-ray intensity of a nearby EGRET source ${ }^{6}$ (3EG 1714-3857) at a location where no actual $\mathrm{GeV}$ flux has been detected (that is, above the background level), although alternative models might reduce the discrepancy (see also ref. 10 ). In addition, the high gas density (about $10^{2}$ protons $\mathrm{cm}^{-3}$ ) needed to explain the $\mathrm{TeV} \gamma$-rays as nucleonic in origin ${ }^{4}$ is simply not compatible - by several orders of magnitude - with the X-ray data ${ }^{7}$ from the same region of the SNR.

As the peaks of both the $\mathrm{TeV}$ (ref. 4) and $\mathrm{X}$-ray (ref. 7) emissions lie in the same lowdensity $^{7}$ region of the remnant (Fig. 1), the most plausible explanation is that they both arise from the same population of relativistic electrons. However, there is an exciting possibility that a minor component of the $\mathrm{TeV}$ emission ${ }^{4}$ could be due to nucleonic

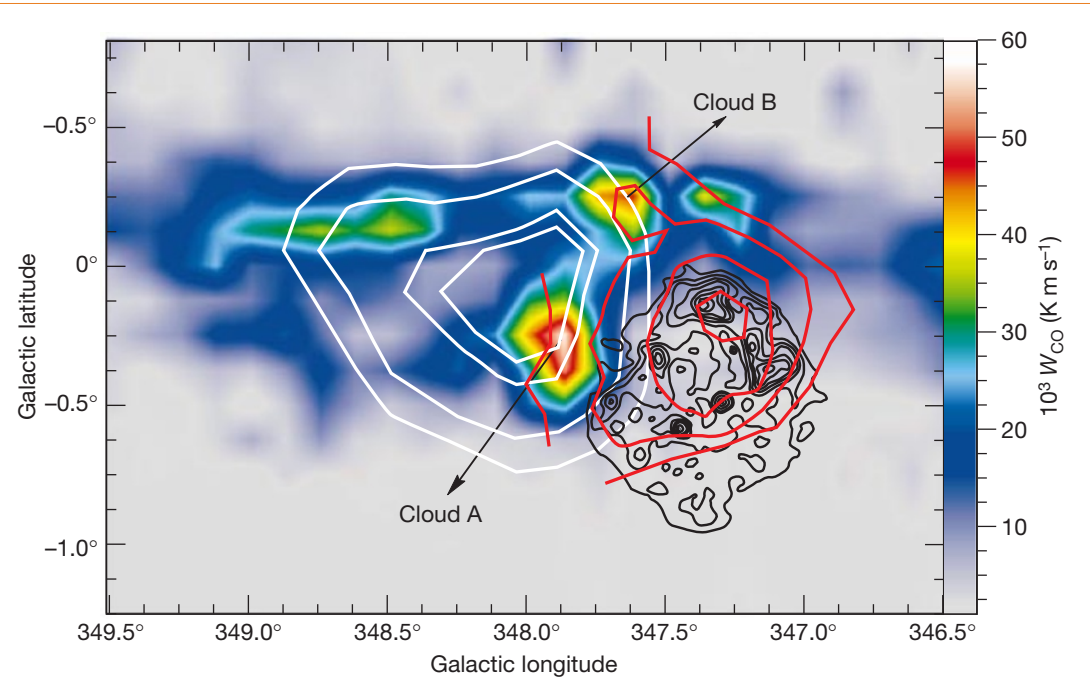

Figure 1 An overlay map in Galactic coordinates showing supernova remnant (SNR) RX J1713.7 - 3946 (G347.3 - 0.5) in dark contours (ROSATPSPC X-ray; from ref. 7). Red, TeV significance contours ${ }^{4}$; white, location-probability contours of the GeV $\left(10^{9} \mathrm{eV}\right)$ EGRET source 3EG J1714 - 3857 (ref. 6); colour scale indicates the intensity of $\mathrm{CO}(J=1 \rightarrow 0)$ emission, and consequently the column density of the ambient molecular clouds, in the velocity interval $v=-105$ to $-80 \mathrm{~km} \mathrm{~s}^{-1}$ associated with the SNR, corresponding to a kinematic distance of $6.3 \pm 0.4 \mathrm{kpc}$ (ref. 5). Note the local maximum of the TeV significance contours at cloud $\mathrm{B}$, which may indicate a hadronic origin of the TeV flux at that location. For further details, see ref. 5 . interactions at the location of the adjacent cloud B (Fig. 1), where sufficient molecular material is present ${ }^{5}$. A suggestive extension and a local maximum in the $\mathrm{TeV}$ significance contours ${ }^{4}$ are coincident with this location.

SNR RX J1713.7 - 3946 might therefore be accelerating nuclei to cosmic-ray energies $^{5}$, but the $\mathrm{TeV} \boldsymbol{\gamma}$-ray signature so far detected from the northwest quadrant of the remnant ${ }^{4}$ is not proof of this. It is likely that most - but not all — of the observed $\mathrm{TeV}$ emission is actually electronic in origin.

To address the question of the source(s) of the $\mathrm{TeV}$ emission properly, better radio data sampled at several frequencies, as well as the inclusion of existing low-energy ROSAT X-ray data, will be needed to determine the primary synchrotron spectrum. Data of greater spatial resolution from the next-generation $\mathrm{GeV}, \mathrm{TeV}$ and neutrino telescopes will also be important. Only when this multifrequency data set is available can the nature of the contributions (nucleonic or electronic) to the observed $\mathrm{TeV}$ emissions start to be disentangled.

Yousaf M. Butt ${ }^{\star}$, Diego F. Torres $\dagger$,

Gustavo E. Romero $\neq$, Thomas M. Dame*, Jorge A. Combił

${ }^{*}$ Harvard-Smithsonian Center for Astrophysics, Cambridge, Massachusetts 02138, USA e-mail:ybutt@cfa.harvard.edu $\dagger$ Department of Physics, Jadwin Hall, Princeton

University, Princeton, New Jersey 08544, USA ¥Instituto Argentino de Radioastronomía, CC 5 , 1894, Villa Elisa, Buenos Aires, Argentina

1. Drury, L. O'C. et al. Space Sci. Rev. 99, 329-352 (2001).

2. Plaga, R. Preprint astro-ph/0111555 at $<\mathrm{http}$ ://xxx.lanl.gov> (2001)

3. Shklovskii, I. S. Dokl. Akad. Nauk SSSR 91, 475-478 (1953).

4. Enomoto, R. et al. (CANGAROO collaboration) Nature 416, 823-826 (2002).

5. Butt, Y. M., Torres, D. F., Combi, J. A., Dame, T. \& Romero, G. E. Astrophys. J. 562, L167-L171 (2001).

6. Hartman, R. C. et al. Astrophys. J. (suppl.) 123, 79-202 (1999).

7. Slane, P. et al. Astrophys. J. 525, 357-367 (1999).

8. Koyama, K. et al. Nature 378, 255-258 (1995).

9. Pohl, M. Astron. Astrophys. 307, L57-L59 (1996)

10. Reimer, O. \& Pohl, M. Preprint astro-ph/0205256 at $<$ http://xxx.lanl.gov> (2002); Astron. Astrophys. (in the press).

COMMUNICATIONS ARISING Behavioural evolution

\section{Does similarity breed cooperation?}

B eciprocity ${ }^{1}$, whether direct ${ }^{2}$ or indirect ${ }^{3}$, is thought to be the key to establishing cooperation among non-relatives. But Riolo et al. ${ }^{4}$ have presented a model in which cooperation is instead based on similarity: agents donate only when their partner's 'tag' lies within a 'tolerance' range around their own. Here we point out that their mode requires individuals with identical tags to cooperate with each other, and show that cooperation tends to collapse when individuals bearing identical tags are given the 
option of not donating. We therefore question their mechanism for maintaining cooperation without reciprocity.

What makes cooperation so challenging for theorists is explaining how it can persist in the face of more exploitative strategies. In the system of Riolo et al., clusters of cooperating agents with similar tags arise intermittently, only to be undermined by agents that reduce their tolerance level, $T$, such that they accept more donations than they offer. However, there is a limit to such cheating imposed by the minimum $T=0$. This means that when individuals with identical tags interact, they must always donate. A striking characteristic of Riolo and colleagues' simulations was the formation, through differential reproduction, of clusters of agents with identical tags. Most individuals in their simulations (up to $97 \%$ of the population ${ }^{4}$ ) were therefore ultimately constrained to cooperate.

To investigate what would happen if agents were given the option of declining to donate to any other agents, even those with identical tags, we replicated Riolo and colleagues' simulations with one simple modification: we allowed tolerance to evolve to below zero. Agents with negative $T$ values would not donate to any other agent, although, by setting the minimum boundary for $T$ at $-10^{-6}$, we ensured that all positive mutational changes converted $T$ back to the cooperative region. We found that introducing the realistic option of non-donation had a catastrophic effect on cooperation (Fig. 1).

Why do we not find the high degree of cooperation reported by Riolo et al.? Once the constraint that identical tags must cooperate has been removed, agents interacting with others bearing the same tag face the classical 'prisoner's dilemma' - they can do well by cooperating, but they can do even better by accepting donations without donating. Thus, mutants that fail to donate, even to those with identical tags, will tend to invade, destroying cooperation.

Cooperation under the original conditions of Riolo et al. operates through a process of 'like helping like ${ }^{5}$ — agents sharing any particular tag also share the rule of donating to each other, so a form of kin selection ${ }^{6}$ can support cooperation. However, agents can have identical tags without having a recent common ancestor, so in our modified system they can share tags without sharing the rule for cooperating. Because tag similarity is no longer a reliable guide to behaviour, the system of 'like helping like' breaks down. Whereas the problematic 'green beard' effect $^{7}$ depends on a link between altruism and a particular trait, the system of Riolo et al. depends on a link between altruism and similarity. Allowing similar individuals not to donate caused cooperation to be restricted in our system, even without any mechanism for cheating through faking tags ${ }^{5}$.

Cooperation based on similarity there-

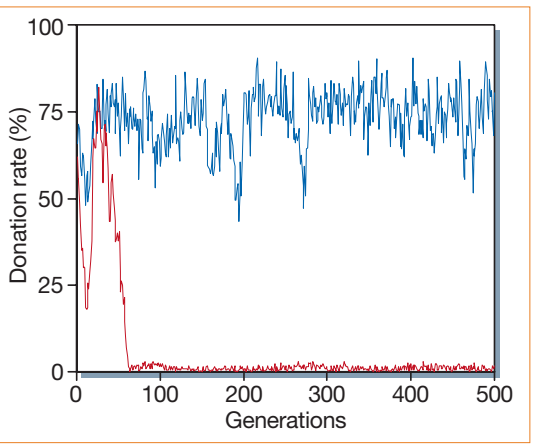

Figure 1 Population dynamics for the first 500 generations of a typical run of Riolo and colleagues' model ${ }^{4}$, in which individuals with identical tags must donate (blue), and our modified model in which individuals with identical tags may or may not donate (red). All parameter values were the same as for Fig. 1 of ref. 4. In 30 runs of our modified model, each for 30,000 generations, the overall mean donation rate was $1.48 \%$ (s.e.m. $0.031 \%$ ), in comparison with Riolo et al.'s $73.6 \%$.

fore turns out to be a rule that was built into the model rather than an inference that can be drawn from it. Nevertheless, we believe that possible mechanisms by which cooperation can arise without reciprocity merit further attention ${ }^{8}$, and the role of signals in such systems will be an important consideration. Gilbert Roberts ${ }^{\star}$, Thomas N. Sherratt $\dagger \neq$ ${ }^{\star}$ Evolution and Behaviour Research Group, School of Biological Sciences, Henry Wellcome Building for Neuroecology, University of Newcastle upon Tyne, Newcastle upon Tyne NE2 4HH, UK

e-mail: gilbert.roberts@ncl.ac.uk

$\uparrow$ School of Biological and Biomedical Sciences, University of Durham, South Road,

Durham DH1 3LE, UK

$\ddagger$ Present address: Department of Biology, Carleton University, Ottawa, Ontario K1S 5B6, Canada

1. Trivers, R. Q. Rev. Biol. 46, 35-57 (1971).

2. Axelrod, R. \& Hamilton, W. D. Science 211, 1390-1396 (1981).

3. Nowak, M. A. \& Sigmund, K. Nature 393, 573-577 (1998).

4. Riolo, R. L., Cohen, M. D. \& Axelrod, R. Nature 414, 441-443 (2001)

5. Sigmund, K. \& Nowak, M. A. Nature 414, 403-405 (2001).

6. Hamilton, W. D. J. Theor. Biol. 7, 1-52 (1964).

7. Dawkins, R. The Selfish Gene (Oxford Univ. Press, New York, 1976).

8. Roberts, G. Proc. R. Soc. Lond. B 265, 427-431 (1998).

Riolo et al. reply - Roberts and Sherratt argue that if agents with identical tags are allowed a choice of behaviour, then tag similarity can no longer be a reliable guide to behaviour and so similarity does not breed cooperation. Although they are correct in noting that in our model ${ }^{1}$ an agent will always donate when it meets another with an identical tag, we do not believe that their basic claim is correct.

We have replicated the results of Roberts and Sherratt and have run a generalized model that includes theirs as one extreme and our original model as another (details are available from R.L.R.). We find that if mutations are not biased as strongly towards 'never donate', as in their version of the model, similarity can indeed breed cooperation. Whether it does, and to what extent, depends on several factors, including the rate at which 'never donate' agents are created, the number of pairings, the cost/benefit ratio of donations and the particular adaptive mechanisms in the model. If unconditional defection is introduced by adding a binary trait that controls whether agents never donate, or donate using tags and tolerance, we find that cooperation also emerges, but again the extent of cooperation depends on many factors.

We believe that the difference has not been fully understood between the stability of cooperation within any particular tag group and the rate of cooperation across a population consisting of diverse tags with changing frequencies over time. There is no dispute that particular cooperative tag groups are invadable ${ }^{1,2}$. However, as one tag group is invaded and thus dies off, another tag group with more reliable cooperators can flourish and become dominant, resulting in the cycles of cooperation and tag dominance noted previously ${ }^{1,3}$.

Roberts and Sherratt claim that cooperation based on similarity was built into our model. It was not, which is why, under some parameter settings (few pairings or high cost of donation), cooperative periods are rare and short-lived, resulting in very low overall donation rates ${ }^{1}$. Nevertheless, the level of cooperation for other parameter settings is substantial, with the overall rate of cooperation depending on the relative dynamics of invasion, resistance and emergence of dominant groups.

Many factors could affect the dynamics generated by tag-based mechanisms. For instance, tags that are easy to copy might lead to high rates of invasion, whereas other tags, such as language or accent, might be difficult to copy ${ }^{3}$. Our model could also be extended to study how a tag mechanism acts in conjunction with other mechanisms known to affect the emergence of cooperation. For example, territorial distribution of agents might favour 'speciation' into selfenforcing stereotypes ${ }^{3}$. Further investigation is needed to understand fully the range of mechanisms that can produce cooperation without reciprocity. Our results show that, under some conditions, tag mechanisms are one viable approach.

Rick L. Riolo*, Michael D. Cohen $\dagger$, Robert Axelrod $\neq$

${ }^{*}$ Center for the Study of Complex Systems, $\uparrow$ School of Information, and $\ddagger$ Gerald $R$. Ford School of Public Policy, University of Michigan, Ann Arbor, Michigan 48109, USA

e-mail: rlriolo@umich.edu

\footnotetext{
1. Riolo, R. L., Cohen, M. D. \& Axelrod, R. Nature 414, 441-443 (2001).

2. Robson, A. J. J. Theor. Biol. 144, 379-396 (1990).

3. Sigmund, K. \& Nowak, M. A. Nature 414, 403-405 (2001).
} 